EPJ Web of Conferences 59, 05008 (2013)

DOI: $10.1051 /$ epjconf/20135905008

(C) Owned by the authors, published by EDP Sciences, 2013

\title{
Laser plasma physics in shock ignition - transition from collisional to collisionless absorption
}

\author{
O. Klimo ${ }^{1,2, a}$, V. T. Tikhonchuk ${ }^{2}$, X. Ribeyre ${ }^{2}$, G. Schurtz ${ }^{2}$, C. Riconda ${ }^{3}$, \\ S. Weber ${ }^{2}$ and J. Limpouch ${ }^{1}$ \\ 1 FNSPE, Czech Technical University in Prague, 11519 Prague, Czech Republic \\ 2 CELIA, University Bordeaux 1 - CNRS - CEA, 33405 Talence, France \\ 3 LULI, University Paris 6 - Ecole Polytechnique - CNRS - CEA, 75252 Paris, France
}

\begin{abstract}
Shock Ignition is considered as a relatively robust and efficient approach to inertial confinement fusion. A strong converging shock, which is used to ignite the fuel, is launched by a high power laser pulse with intensity in the range of $10^{15}-10^{16} \mathrm{~W} / \mathrm{cm}^{2}$ (at the wavelength of $351 \mathrm{~nm}$ ). In the lower end of this intensity range the interaction is dominated by collisions while the parametric instabilities are playing a secondary role. This is manifested in a relatively weak reflectivity and efficient electron heating. The interaction is dominated by collective effects at the upper edge of the intensity range. The stimulated Brillouin and Raman scattering (SBS and SRS respectively) take place in a less dense plasma and cavitation provides an efficient collisionless absorption mechanism. The transition from collisional to collisionless absorption in laser plasma interactions at higher intensities is studied here with the help of large scale one-dimensional Particle-in-Cell (PIC) simulations. The relation between the collisional and collisionless processes is manifested in the energy spectrum of electrons transporting the absorbed laser energy and in the spectrum of the reflected laser light.
\end{abstract}

\section{INTRODUCTION}

In the Shock Ignition (SI) scheme [1] the fuel assembly and ignition phases are separated. The ignition conditions are achieved due to an extra shock wave, which is launched by a high power laser spike with the intensity in the range of $10^{15}-10^{16} \mathrm{~W} / \mathrm{cm}^{2}$ (at the wavelength $351 \mathrm{~nm}$ ) and which runs into the preassembled fuel. At the upper side of this intensity range nonlinear kinetic processes start play an important role. The transition from collisional- (lower intensity domain) to collisionless-dominated (higher intensity domain) laser absorption is studied in this paper using fully kinetic collisional PIC simulations in one-dimensional geometry. The code includes high order particle shapes, specially designed boundary conditions and relativistic Coulomb collisions.

The spatial plasma profiles calculated in hydrodynamic simulations [2] of recent experiments [3] are used as initial conditions. They define the approximate conditions in plasma corona at the time of the laser spike arrival. The electron temperature is in the range $1.7-1.9 \mathrm{keV}$, the ion temperature $0.5-$ $1.1 \mathrm{keV}$, the plasma Mach number is ranging from about 1 to 3 , the density profile is approximately exponential with the scale length $L=320 \mu \mathrm{m}$.

The simulation time is sufficiently long (up to $100 \mathrm{ps}$ ) to achieve a quasi-stationary interaction regime. The laser pulse intensity is $1,2.4$ or $8 \times 10^{15} \mathrm{~W} / \mathrm{cm}^{2}$ and the pulse has a constant intensity with a 5 ps linear ramp at the beginning. The ion charge and mass are 3.5 and 7 times the proton mass

\footnotetext{
${ }^{a}$ e-mail: ondrej.klimo@fjfi.cvut.cz
}

This is an Open Access article distributed under the terms of the Creative Commons Attribution License 2.0, which permits unrestricted use, distribution, and reproduction in any medium, provided the original work is properly cited. 

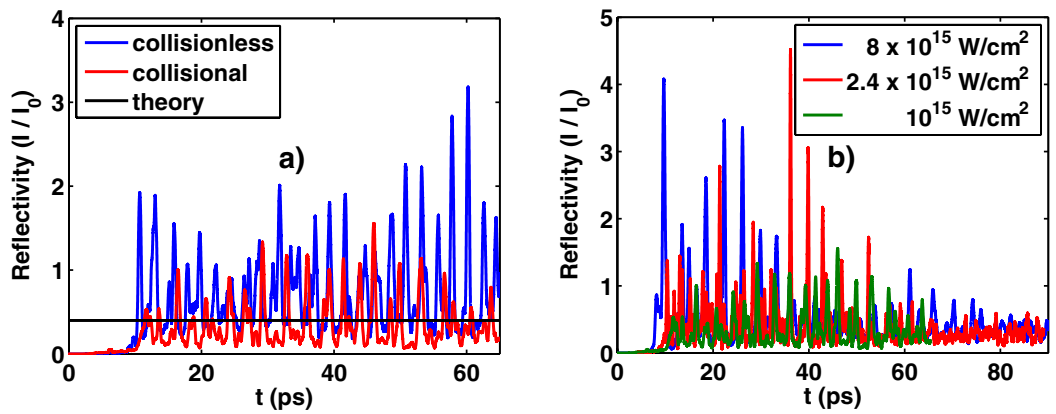

Figure 1. The temporal evolution of the reflectivity (a) in the collisional and the collisionless simulation of laser plasma interaction at the intensity $10^{15} \mathrm{~W} / \mathrm{cm}^{2}$, (b) and in the collisional simulations of laser plasma interaction with the laser pulse intensity $1,2.4$ and $8 \times 10^{15} \mathrm{~W} / \mathrm{cm}^{2}$. The reflectivity related to the inverse Bremsstrahlung absorption is included for comparison in (a).

respectively (fully ionized deuterated plastic CD). The collision frequency is calculated with constant Coulomb logarithm 10.

\section{SIMULATION RESULTS AND DISCUSSION}

The temporal behavior of reflectivity is presented in Fig. 1. Two runs for the lower laser intensity $10^{15} \mathrm{~W} / \mathrm{cm}^{2}$ - collisionless and with collisions included - are compared in panel a) with an estimate from the theoretical collisional absorption model [4]. The overall reflectivity in the collisionless case is more than $60 \%$ and the reflected light comes in a series of intense bunches that originate from the convectively amplified SBS waves. In the collisional case, the bunches of the reflected light are much less intense and the overall reflectivity is little less than $30 \%$, which is expected from the linear theory of inverse bremsstrahlung absorption.

The reflectivity in the SRS frequency range ( $\omega<0.9 \omega_{0}$, where $\omega_{0}$ is the laser frequency) is below $5 \%$ level in the collisional simulation, while it is about $13 \%$ in the collisionless case. If collisions are not taken into account, the scattering instabilities are thus much stronger and SRS may even enter into the self-organized regime. This regime has been studied in [5] for the case of a higher temperature $\left(T_{e}=5 \mathrm{keV}\right)$, same scale $(L=300 \mu \mathrm{m})$ plasma irradiated by the laser pulse with the irradiance of $1.2 \times 10^{15} \mathrm{~W} / \mathrm{cm}^{2} \times \mu \mathrm{m}^{2}$. It has been demonstrated that the laser energy is absorbed in density cavities, which are created and maintained by two coupled SRS processes forming a self-organized resonator between the zones of 1/4th and 1/16th of the critical density. This plasma response was attributed to the high initial plasma temperature, which results in strong Landau damping of SRS induced electron plasma waves with exception of the resonant points, where SRS is growing as an absolute parametric instability. These collective processes are suppressed at this relatively low intensity due to Coulomb collisions.

The importance of parametric processes increases at higher laser intensities. We demonstrate that by changing the laser pulse intensity to 2.4 and $8 \times 10^{15} \mathrm{~W} / \mathrm{cm}^{2}$, while keeping the same initial temperature and density profiles. As it is shown in Fig. 1 (b), the reflectivity in both cases saturates at the level of about $35 \%$. If the laser pulse is much longer than the transient stage taking $30-50 \mathrm{ps}$, about $65 \%$ of its energy can absorbed in the target. For the higher intensity, this absorption is mostly due to a cascade of two SRS processes as described above.

The spectra of the temporally integrated reflected light shown in Fig. 2(a) demonstrate similar features in the frequency range $0.5 \omega_{0}-\omega_{0}$. The narrow signal around $\omega_{0}$ is due to SBS. The broad spectrum around $0.7 \omega_{0}$ comes from the convective SRS in the lower density plasma around $0.1 n_{c}\left(n_{c}\right.$ being the critical density). The strong signal around $0.5 \omega_{0}$ is due to absolute SRS at quarter critical 

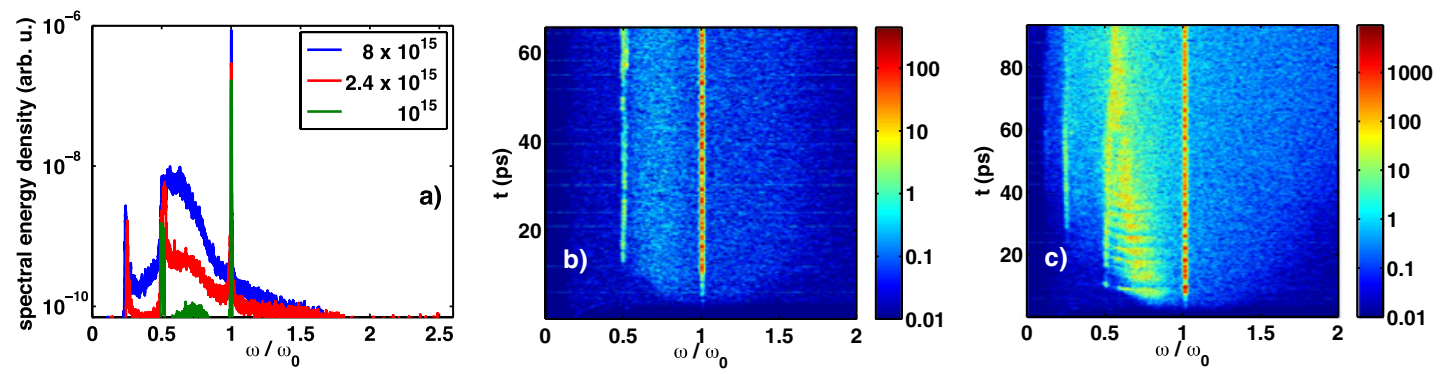

Figure 2. The temporally integrated spectrum of the reflected light in the collisional simulations of laser plasma interaction with the laser pulse intensity $1,2.4$ and $8 \times 10^{15} \mathrm{~W} / \mathrm{cm}^{2}$ is shown in panel (a). The temporal evolution of the spectra of the reflected light in the collisional simulation with the intensity $10^{15} \mathrm{~W} / \mathrm{cm}^{2}$ (b) and the intensity $8 \times 10^{15} \mathrm{~W} / \mathrm{cm}^{2}(\mathrm{c})$.

density. In the case of higher laser pulse intensities, a strong signal at $0.25 \omega_{0}$ is observed too. It comes from the secondary SRS process induced at 1/16th of critical density.

The spectra show us the repartition of energy between different parametric processes. Integrating the spectral energy density we find that the spectrum corresponding to SRS $\left(\omega<0.9 \omega_{0}\right)$ contains about 6,10 and $26 \%$ of the total energy of the reflected pulse for the intensities increasing from 1 through 2.4 to $8 \times 10^{15} \mathrm{~W} / \mathrm{cm}^{2}$ respectively. The relative importance of SRS in comparison with SBS grows with the laser pulse intensity.

The temporally resolved spectra of reflected light for the lowest and the highest intensity are presented in Fig. 2 (b) and (c) respectively. The SRS spectra in the high intensity case (c) shows a fringe like structure in the frequency range around $0.7 \omega_{0}$. The fringes are oblique and the higher frequency part is coming first. This indicates that the amplification of the scattered waves starts in a less dense plasma and the waves propagate in the forward (with respect to the incident laser pulse) direction. This is thus a clear indication of the convective forward SRS. Indeed, the forward SRS is not strongly suppressed in high temperature plasma by Landau damping. The forward SRS light waves have also been confirmed in our simulations by monitoring the spectrally resolved light penetrating behind the quarter critical surface.

In these simulations the forward SRS is not the dominant mechanism and it is suppressed by the cavities, which develop in the lower density plasma. The plasma cavitation is due to the rescattering of the Raman scattered light, which is responsible for the signal at $0.25 \omega_{0}$. Therefore, the fringes in the SRS spectra start to disappear when the signal at $0.25 \omega_{0}$ appears. We observe that the importance of forward SRS increases with the laser pulse intensity. Thus it deserves further studies also in the context of SI and it will be subject to our forthcoming publication.

The energy distribution of electrons in the simulation box in the initial and final time (64 ps) are essentially Maxwellian with temperatures $2 \mathrm{keV}$ and $2.5 \mathrm{keV}$ in the collisional simulation with the intensity $10^{15} \mathrm{~W} / \mathrm{cm}^{2}$, Fig. 3(a). This clearly demonstrates efficient heating of the bulk population of electrons due to the collisional absorption process. Subtracting the initial energy distribution from the final one and integrating, we find the amount of absorbed energy, which is contained in thermal electrons. It is about $60 \%$ of the absorbed laser pulse energy. The rest of the absorbed energy is contained in accelerated ions (about $20 \%$ ) or has already been transported to the target rear side by higher velocity $(v \approx 0.2-0.3 c$, i.e. $E \approx 10-30 \mathrm{keV}$ ) electrons (about $20 \%$ ).

It follows from the spectra of reflected light in Fig. 2(a) that the repartition between the collisional and collisionless processes changes with the laser intensity. The differential (in energy) electron flux in Fig. 3(b) shows high energy tail corresponding to Maxwellian electron energy distribution with the temperature of about $23 \mathrm{keV}$ for the higher laser intensities 2.4 and $8 \times 10^{15} \mathrm{~W} / \mathrm{cm}^{2}$. The temperature of these hot electrons does not change significantly, while the number of hot electrons increases with the 

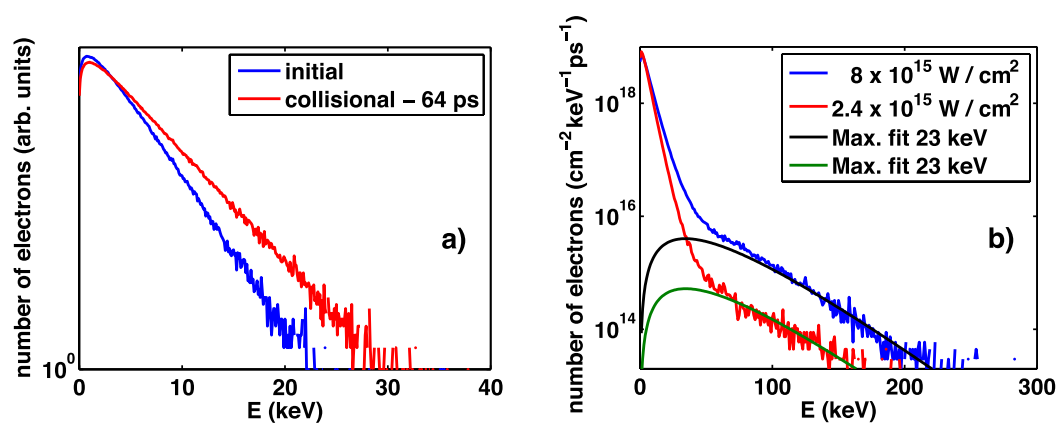

Figure 3. (a) The electron energy distributions in the simulation box at the beginning and at the end of the collisional simulation with the laser pulse intensity $10^{15} \mathrm{~W} / \mathrm{cm}^{2}$. (b) The temporally averaged flux of electrons flying into the target differential in energy recorded inside the target behind $1 / 4 n_{c}$ in the collisional simulation. The differential electron flux is related to the distribution function and the fits of the high energy tails with Maxwellian functions can be performed.

laser intensity. From the energy distributions of electrons we find that the fraction of energy absorbed by collisionless processes increases from 5 to 70 and $93 \%$ raising the laser pulse intensity from 1 to 2.4 and than to $8 \times 10^{15} \mathrm{~W} / \mathrm{cm}^{2}$.

There are virtually no hot electrons at the lowest laser intensity, while at higher intensities an increasing fraction of the absorbed energy is transported into the target with the electrons having the average energy of about $40 \mathrm{keV}$ (maximum of the function $E \Psi(E)$, where $\Psi(E)$ is the differential (in energy) electron flux). The hot electrons $(\mathrm{E}>30 \mathrm{keV})$ carry about $62 \%$ of the incident laser pulse energy in the case of the highest intensity. For the laser pulse intensity $2.4 \times 10^{15} \mathrm{~W} / \mathrm{cm}^{2}$, the energy carried by hot electrons $(\mathrm{E}>30 \mathrm{keV})$ is about $29 \%$.

\section{CONCLUSIONS}

In conclusion, in the lower end of the studied intensity range, the electron collisions largely suppress the SBS and about $70 \%$ of the laser pulse energy is absorbed. The SRS reflectivity is below 5\% and thus about $65 \%$ of laser energy are absorbed directly due to electron-ion collisions. At the upper boundary of the intensity domain, the collective effects dominate. The reflectivity remains at the level about $35 \%$ and the energy absorption occurs in the density cavities developing in the resonance zones near 1/4th and 1/16th of the critical density. The forward convective SRS is observed and it may become and important process for higher laser pulse intensities, but it play only secondary role in the simulations of this paper. It will be studied in more details in our forthcoming publication. The plasma reflectivities and the hot electrons are in a relatively good agreement with the results measured at the Omega laser [3] (i.e. the measured reflectivity in the high intensity domain of $36 \%$ and the hot electron temperature of about $40 \mathrm{keV}$ ). The comparison with the measured SRS spectra and the number of hot electrons are less satisfactory (the SRS spectrum is dominated by convective SRS coming from lower density plasma and the hot electrons carry about $10 \%$ of the laser energy in the experiment) and they deserve more studies.

This work was granted access to the HPC resources of CINES under the allocation 2011-056129 made by GENCI (Grand Equipement National de Calcul Intensif). The support by the Czech Science Foundation, project P205/11/P660 by the Czech Ministry of Education, Youth and Sports (projects LC528 and MSM6840770022) are acknowledged. This work was completed within the European project HiPER, the support of Euratom is gratefully acknowledged. 


\section{IFSA 2011}

\section{References}

[1] R. Betti, C. D. Zhou, K. S. Anderson, L. J. Perkins, W. Theobald, and A. A. Solodov, Phys. Rev. Lett. 98, 155001 (2007)

[2] X. Ribeyre and M. Lafon, private communication concerning hydrodynamic simulations of Omega experiments [3] (2010)

[3] W. Theobald, R. Betti, K. S. Anderson, O. V. Gotchev, D. D. Meyerhofer, C. Ren, A. A. Solodov, C. Stoeckl, V. A. Smalyuk, J. A. Delettrez, V. Yu. Glebov, F. J. Marshall, J. F. Myatt, T. C. Sangster, W. Seka, B. Yaakobi, J. A. Frenje and R. D. Petrasso, LLE Review, Quarterly Report 119, 117 (2009)

[4] S. Eliezer, Interaction of High Power Lasers with Plasmas (Institute of Physics Publishing, Bristol, 2002)

[5] O. Klimo, S. Weber, V. T. Tikhonchuk and J. Limpouch, Plasma Phys. Contr. Fusion, 52, 055013 (2010) 